LA-7600-MS

Informal Report

Special Distribution

Issued : January 1979

\title{
A Study of Relativistic Electron Beams Generated by a Foilless Diode
}

Michael E. Jones

Lester E. Thode

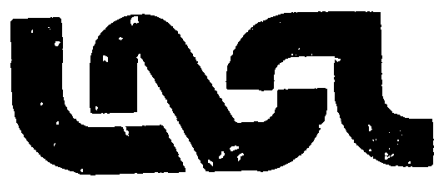




\title{
A STUDY OF RELATIVISTIC ELECTRON BEAMS GENERATED BY A FOILLESS DIODE
}

by

Michael E. Jones and Lester E. Thode

\begin{abstract}
ABSTRATT
Preliminary results of a numerical and analytical study of foilless diodes are presented. The work has produced an electron emission algorithm for the particlein-cell simulation code CCUBE. Diode performance has been studied as a function of applied magnetic field strength and simple geometry changes. Annular electron beams with an energy of $5 \mathrm{MeV}$ appear obtainable with densities exceeding $10^{14} \mathrm{~cm}^{-3}$.
\end{abstract}

\section{I . INTRODUCTION}

A theoretical investigation into the generation of intense relativistic electron beams by foilless diodes is being conducted at Los Alamos Scientific Laboratory (LASL) under contract to the Air Force Office of Scientific Research (AFOSR). The goal of the study is to determine the feasibility of producing a 5- to $50-\mathrm{MeV}$ electron beam with a current density of 50 to $500 \mathrm{kA} / \mathrm{cm}^{2}$ for use in several applications including, submillimeter microwave generation, high-density plasme heating, and collective ion acceleration. ${ }^{1}$ The applications require that the beam produced be laminar and, consequently, nonthermal. It is also desirable for some of the applications to produce an annular beam.

Much of the investigation consists of numerical simulations of the foilless diode. Consequently, the first part of work has involved computer code development. An existing particle-in-cell code, CCUBE, ${ }^{2-4}$ has been modified to include an electron emission algorithm, and a series of preliminary simulations of foilless diodes have been performed.

The simulations have included parameter studies of magnetic field and geometry factors. The results, though not fully analyzed yet, indicate that 
the emission from the shank of the cathode may not be as thermal as has been expected from experiments on magnetically insulated transmission lines. The total current emitted by the diode appears to be limited by the impedance of the coaxial transmission line. The beams that have been produced so far have been somewhat thermal for most applications and the kinetic energy of the beams is below the applied voltage on the diode. Both of these results appear to be related to beam equilibrium conditions. A proper choice of geometry and magnetic field should allow one to tailor the equilibrium of the beam to meet the needs of the applications listed above. The simulations show promise for this prospect.

There has been some collaboration with the Air Force Weapons Laboratury's (AFWL) experimental program which has shown agreement between the foilless diode simulations and experiments of a similar nature. ${ }^{5}$ Closer collaboration in this area is planned for the future.

\section{THE EMISSION ALGORITHM}

The numerical simulation studies of the foilless diode have been performed using a fully relativistic, electromagnetic, space- and time-dependent, twodimensional particle-in-cell computer code (CCUBE). Much of the effort Lo date "in the foilless diode study has been spent developing and improving an emission algorithm for CCUBE.

Electrori emission from the surface of the cathode is assumed to be spacecharge limited. This gives the boundary condition that emission must occur at a sufficient rate to force the normal component of the electric field to zcro at the surface of the cathode. The algorithm allows emission only after an electric ficld threshold has been reached on the cathode surface, which allows the modeling of different cathode materials. Because of the finite difference technique used by CCUBE, only the tangential components of the electric field are known on the surface of the cathode. The normal component is found one-half cell away. Thus, the space-ciarge limited boundary condition is approximated by minimizing the normal electric field at the half cell location. Since CCUBE uses nearest grid point weighting in the normal direction, this means the minimum electric field must be large enough to give a particle emitted from the cathode a gyroradius of at least one cell. In the dimensionless units of the code the minimum field $E_{m i n}$ is found to be 


$$
E_{\min }=\operatorname{RDX}-\left(\mathrm{RDX}^{2}+\mathrm{B}^{2}\right)^{1 / 2}
$$

where $\mathrm{RDX}$ is the reciprocal of the cell width and $\mathrm{B}$ is the tangential magnetic field in the first cell outside the cathode. The boundary condition is implemented by emicting a sufficient number of particles from the cathode to satisfy Poisson's equation at the surface. The particles are given only a small thermal velocity. The normal field accelerates the particles producing a current, which through Maxwell's equations reduces the electric field. Particles are emitted between cell points without recourse to elaborate weighting and interpolation methods. Any error in the amount of charge emitted is self-correcting, and the code has been run several thousand time steps without appreciable noise buildup.

\section{THE SIMULATIONS}

The foilless diode simulations solve a class of problems which scale with the time scale. One problem simulated corresponds to parameters which can be realized experimentally by the relativistic electron beam device which is to be obtained by LASL. Specifically, the parameters of the simulations have included a 5-MV voltage pusse which is launched onto a 37-ohm transmission line leading to the diode. ${ }^{6}$ In its simplest form the foilless diode consists of a coaxial transmission line with a truncated center conductor as the cathode of the diode. Electrons leave the cathode by field emission. An axial magnetic field of sufficient strength prevents the electrons from striking the outer conductor. The simulations have modeled this type of diode for a center conductor (cathode) radius of $1 \mathrm{~cm}$ and an outer conductor (anode) radius of $1.85 \mathrm{~cm}$ for a variety of magnetic field strengths. Also, a series of simulations was performed for slightly more complicated geometry, in which the anode radius is abruptly reduced from $1.85 \mathrm{~cm}$ to $1.13 \mathrm{~cm}$. But only one simulation has been run to determine the effect of cathode shaping on diode performance.

All the simulations were performed in a cylindrical, r-z, coordinate system. The boundary conditions used require the tangential electric fields be zero and particles be absorbed on the conductors. The lower $z$ boundary condition launches the TEM wave with a $0.5-n s e c$ risetime, while the condition on the upper $z$ boundary transmits the wave and particles. The first 10 cells in the $z$-direction along the cathode are not emitting in order to allow the IEM wave to become established, while the rest of the cathode emits space-charge limited 
electron current after the electric field exceeds $800 \mathrm{kV} / \mathrm{cm}$. A grid which contains 56 cells in the $r$-direction and 100 cells in the $z$-direction was found to be adequate for the foilless diode simulations. The number of particles varies dynamically during a run. The voltage pulse remains on long compared to the risetime and an equilibrium is established which has between 10 and 30 thousand particles depending on the particular run.

\section{RESULTS AND INTERPRETATION}

A. Magnetic field effects

A series of simulations was performed for the simple truncated-coax diode in order to determine the effects of applied axial magnetic field strength. Figure 1 shows the total current transmitted out of the diode as a function of magnetic field, obtained from the simulations. For small magnetic field strengths the electrons are not confined and current is lost to the anode wall. as shown in the particle plots of Fig. 2. The current increases with magnetic field strength to a maximum at intermediate fields then decreases for large field strengths. In no case was the total current larger than the $135 \mathrm{kA}$ which would be obtained if the impedance of the diode were the same as the vacuum impedance of the transmission line, that is, $37 \mathrm{ohms}$.

This kind of current dependence on magnetic field strength agrees qualitatively with experiments performed at AFWL. Figure 3 shows the results of one of these experiments. Note the current exhibits the same increase with magnetic field strength, reaches a maximum, and is reduced at large magnetic fields. Since the parameters of the AFWL experiment are different from those of the simulation, the comparison is only qualitative. The experiment was performed with a voltage of $1.5 \mathrm{MV}$, an anode radius of $2.4 \mathrm{~cm}$ and a cathode radius of $1.32 \mathrm{~cm}$.

As can be seen in Fig. 2, the electron beam expands as it moves away from the cathode. The amount of expansion depends on the magnetic field strength. The simulations indicate that the beam is hollow and the inside radius depends only weakly on magnetic field strength. A calculation of the space-charge limited current as a function of outside beam radius for a $5-\mathrm{MeV}$ hollow beam with an inside radius of $0.9 \mathrm{~cm}$ propagating in a grounded conducting tube with a radius of $1.85 \mathrm{~cm}$ is shown in Fig. $4 .^{7}$ The graph shows that the space-charge limited current depends strongly on beam radius. For large beam radii (small magnetic fields) the space-charge limit greatly exceeds the current observed in 
the simulations. As the radius of the beam is reduced (larger magnetic fields), the currents observed in the simulations are very nearly equal to the calculated space-charge limit. Thus the electron current in the diode appears limited by the transmission line impedance for small magnetic field strengths and limited by space-charge for strong fields.

B. Equilibrium Model

An understanding of beam equilibria is important for several reasons. Firstiy, matching the impedance of the beam to the impedance of the rest of the diode should result in maximum power transfer. Secondly, because the equilibrium involves a radially dependent particle energy, the beam is subject to velocity shear instabilities, notably the Diocotron instability, which can disrupt the propagatiun of the beam. Finally, knowledge of beam equilibria properties will aid in the design of nonthermal beams.

The particle plots of Fig. 2 indicate that the beam expands in the weaker magnetic fields in order to attain an equilibrium. It is desirable to know how this equilibrium changes with magnetic field strength. Thus a simple analytical model was used to gain insight into the equilibrium of hollow electron heams.

It is assumed that the beam is emitted uniformly from an annular region on the cathode. Electrons are emitted with zero initial velocity and the beam expands radially to an equilibrium. The equilibrium thus formed has no axial or azjmuthal dependence. The beam is treated as a relativistic cold fluid, and Maxwell's equations along with conservation of energy and canonical angular momentum form a closed set of equations describing the equilibrium. Explicitly, the equations to be solved are

$$
\begin{aligned}
& \gamma m v_{\theta}^{2} / r=e\left\{E_{r}+\left[v_{\theta}\left(B_{z}+B_{0}\right)-v_{z} B_{\theta}\right] / c\right\} \quad \text { (force balance), } \\
& \left.\begin{array}{l}
d B_{z} / d r=4 \pi e n v_{\theta} / c \\
1 / r d\left(r B_{\theta}\right) / d r=4 \pi e n v_{z} / c
\end{array}\right\} \begin{array}{c}
\text { (Maxwell's equations) , } \\
1 / r d\left(r E_{r}\right) / d r=-4 \pi n e
\end{array} \\
& \gamma(r)=\gamma_{0}+e / m c^{2} \int_{r}^{c} E_{r} d r \quad \text { (conservation of energy), }
\end{aligned}
$$




$$
\gamma m r V_{\theta}=e / c \int_{0}^{r}\left(B_{z}+B_{0}\right) r^{\prime} d r^{-}-e B_{0} r_{o}^{2} / 2 c \quad \text { (canonical angular momentum), }
$$

and

$$
n r d r=n_{0} r_{0} d r_{0} \quad \text { (conservation of electron number), }
$$

where $e$ and $m$ are the charge and mass of the electron respectively. The oniy nonzero fluid variables are the density $n$, the axial fluid velocity $V$, and azimuthal fluid velocicy $V_{\theta}$. The nonzero field variables are the radial electric field $E_{r}$, the azimuthal magnetic field $B_{\theta}$, and the axial magnetic field which is written as $B_{0}$, the applied field, plus $\mathbf{S}_{2}$, the self-consistent $f$ ield. The relativistic factor that the electrons would obtain by moving from the cathode to the anode is denciced by $\gamma_{0}$ and is determined by the applied voltagr. The position of a streamline is denoted by $r$, and $r$ denotes the radial position on the cathode surface from which the streamline originates. The anode radius is $R_{C}$.

When $B_{z}$ is small compared to $B_{o}$ and $f_{n}$ the limit $V_{z} \rightarrow c$, we find the following expression for the applied magnetic field necessary for equilibrium,

$$
\mathrm{B}_{\mathrm{o}}^{2}=-\mathrm{b} \pm\left(\mathrm{b}^{2}-\mathrm{a}^{2}\right)^{1 / 2}
$$

where

$$
\begin{aligned}
& b=8 I^{2} R^{2} / c^{2}\left(R^{2}+R_{o}^{2}\right)^{2}-8 I R_{p}^{2} \gamma_{e} m c / e\left(R^{4}-R_{o}^{4}\right), \\
& a=4 I R^{2} m c / e\left(R^{4}-R_{o}^{4}\right),
\end{aligned}
$$

and

$$
\gamma_{e}=\gamma_{o}-2 e I / m c^{3} \ln \left(R_{c} / R\right)
$$

Here, $R$ is the outer radius of the beam, $R_{0}$ is the cathode radius, I is the total current, and $\gamma_{e}$ is the relativistic factor at the beam edge. The magnetic field necessary for equilibrium is independent of beam thickness and hence 
independent of current density. The expression may be inverted to obtain the outer radius of the beam as a function magnetic field for a fixed current and voltage as shown in Fig. 5 .

When compared to the simulation results the expression overestimates the minimum applied magnetic field necessary to confine the beam by $50 \%$. However, for larger magnetic fields the predicted radius agrees to within $5 \%$ of that obtained in the simulation. The assumption that the beam expands uniformly probably is only valid if the beam expands a small fraction of its original radius, that is, the cathode radius. Thus, for beam radii only slightly larger than the cathode radius the model may be used to obtain an expression for the beam impedance which we define as the anode-cathode voltage divided by the beam current. An example of the beam impedance as a function of magnetic field calculated in this way is shown in Fig. 6.

The model presented here can be improved to better describe the diode operation. There is a definite axial dependence in the beam equilibrium near the cathode, the effects of which are propagated down the beam. The simulations indicate that the bean equilibrium might not be steady state, but might possess a standing wave character. This effect will be the subject of further study. C. Geometric Effects

A series of simulations were performed in a slightly more complicated geometry in which the anode radius changes abruptly from $1.85 \mathrm{~cm}$ to $1.13 \mathrm{~cm}$. The distance from the end of the cathode to the reduced anode radius was varied, and the resulting currents are shown in Fig. 7. The solid circle corresponds to a shaped cathode. The electrode geometry and corresponding particle ilots are shown in Fig. 8. The currents generated are lower than those obtained in the simple truncated-coax diode.

The diode performance changes dramatically for only small changes in geometry. In some cases the beam expands and strikes the anode, while for only a slight geometry change the beam pinches. It was found that the particles striking the anode do not obtain the full kinetic energy of the applied voltage. This suggests that the TEM pulse is reflected and the equilibrium is a form of standing wave which prevents the anode from reaching the full 5-MV potential. The simulation results are consistent with the equilibrium model only if one assumes the anode-cathode potential difierence is much less than $5 \mathrm{MV}$. A small amount of shaping of the cathode greatly increases the diode current. Thus it. 
appears that diode impedance depends on geometry in a complex way, and it is not clear that increasing the complexity of the geometry improves the performance of the diode.

\section{Shank Emission}

In all simulations thus far the cathode shank appears to be magnetically insulated for applied magnetic field strengths which reasonably confine the beam. The results are consistent with simple theoretical estimates of the shank emission properties. A sheath forms on the shank which has an electron density of $\sim 10^{14} \mathrm{~cm}^{-3}$. Calculation and simulation indicate that the electron temperature should not greatly exceec the emission temperature, which is taken conservatively to be $25 \mathrm{eV}$. Becaust; of the influence of the applied magnetic field as well as the azimuthal self-magnetic field of the beam the electrons are deflecied back to the cathode before they have a chance to gain very much energy in the electric field. Thus, it appears that the electrons which enter the beam from the sheath do not contribute signifirantly to the temperature of the beam.

\section{SUMMARY}

The research effort so far has produced an emission algorithm for the relativistic, electromagnetic, time-dependent particle-in-cell simulation code CCUBE. Preliminary simulations of the foilless diode have scudied the effect of applied magnetic field strength and simple diode geometry changes. For small magnetic field strengths, the diode current is limited by the impedance of the transmission line supplying power to the diode. At large magnetic field strengths, the electron beam is confined to a radius sufficiently small compared to the anode radius that the diode current which can propagate down the hollow anode is limited by space-charge effects. The impedance of the diode depends strongly on minor geometry changes in a complex way, and indications are that increasing the complexity of the geometry of the diode without careful study may be more of a detriment than an improvement to diode performance.

Some analytical modeling of the beam equilibrium has been done. An expression for the beam impedance has been obtained which should prove useful for the diode design.

The temperature of the beams produced in the simulations so far has not been measured accurately, but the indications are that, $\delta \gamma / \gamma^{3}$ may be as small as $10^{-3}$ at the exit of the diode. The simulations show promise for reducing the beam temperature further. No simulations have been performed using adiabatic 
compression yet. However, beam densities of up to $5 \times 10^{13} \mathrm{~cm}^{-3}$ have been obtained, and densities of $10^{14}$ to $10^{15} \mathrm{~cm}^{-3}$ appear obtainable without compression.

\section{ACKNOWLEDGMENTS}

The authors would like to acknowledge enlightening discussions with C. Clark and D. Straw. We are particularly indebted to B. Godfrey for many helpful suggestions.

\section{REFERENCES}

1. Lester E. Thode, "A Proposal for the Study of Vacuum Adiabatic Compression of a Relativistic Electron Ream Generated by a Foilless Diode," Los Alamos Scientific Laboratory report LA-7169-P (February 1978).

2. B. B. Godfrey and L. E. Thode, "Galerkin Difference Schemes for Plasma Simulation Codes," Proc. 7th Conf. Num. Sim. Plas., New York, 1975 p. $87-91$.

3. B. B. Godfrey, "Application of Galerkin's Method to Particle-ln-Cell Plastna Simulation," Proc. 8th Conf. Num. Sim. Plas., Monterey, 1978, Paper PE-3.

4. B. B. Godfrey, "A Galerkin Algorithm for Multi-l)imensional Plasma Simulation Codes," Bull. Am. ihys. Soc. 23, 841 (1978).

5. 1). C. Straw and M. C. Clark, "Foilless Diode Behavior in a Strong External Magnetic F'ield," Bull. An. Phys. Soc. 23, 839 (1978).

6. Michael E. Jones and Lester E. Thode, "Numerical Simulations of Coaxial Foilless Diodes," Bull. Am. Phys. Soc. 23, 839 (1978).

7. T. C. Genoni and W. A. Proctor, "Annular Electron Beam Propagation in an Evacuated, Equipotential Cylinder at Relativistic Energies in the Steady State, Strong Magnetic Guide Field Limit," in IEF. Conference Record - The 1978 IEEE International Conference on Plasma Science, Monterey, California, 


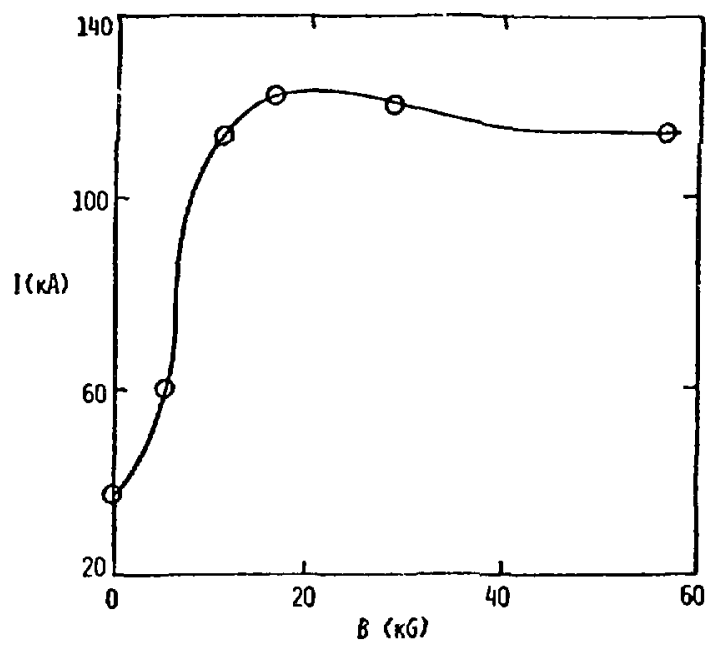

Fig. 1. Diode current as a function of applied magnetic field strength.

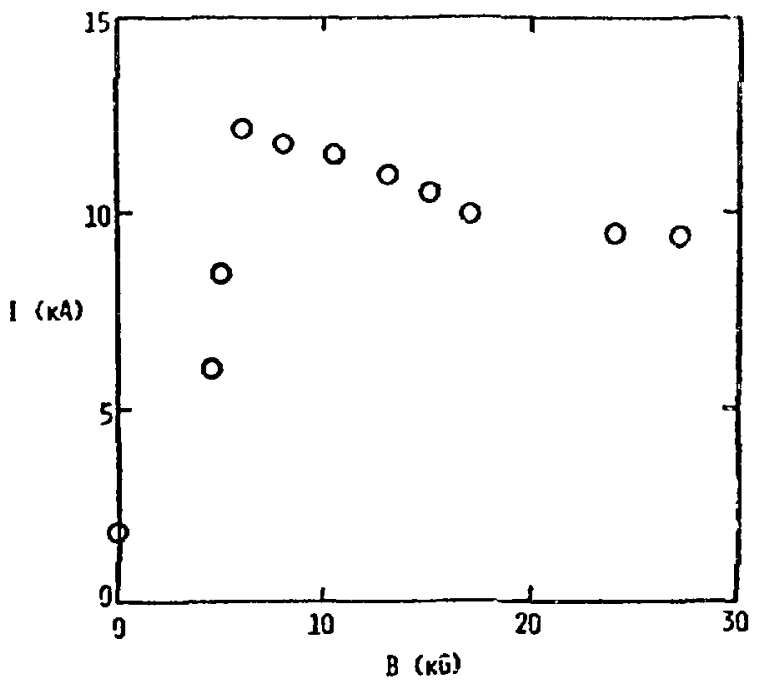

Fig. 3. AFWL experimental results for diode current versus applied magnetic fieid strength.

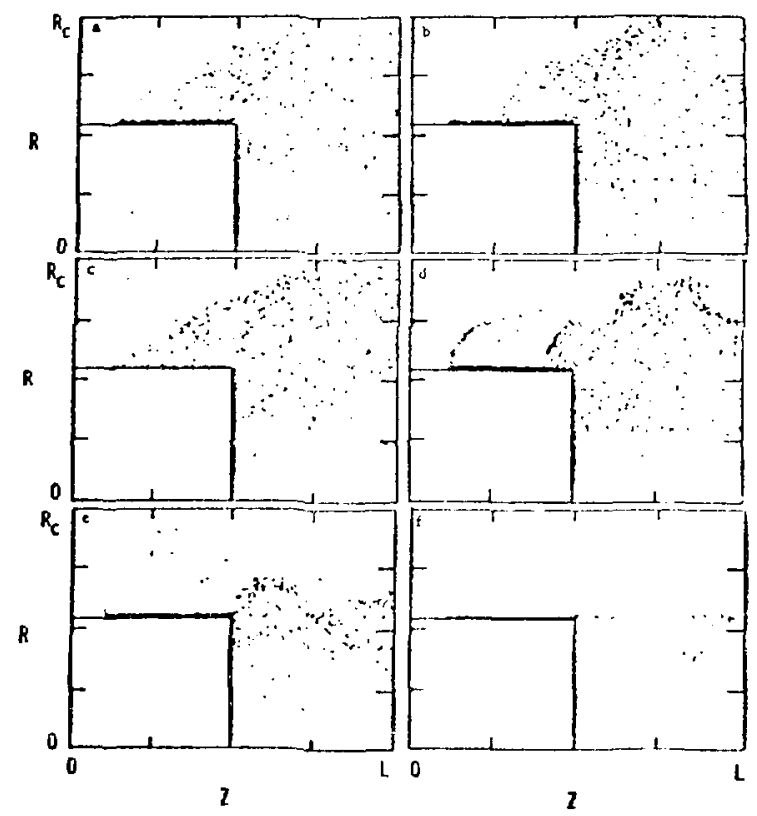

Fig. 2. Equilibrium particle maps for various applied magnetic: fields. (a) $B=0$,

(b) $B=5.7 \mathrm{kG}$, (c) $B=11.4 \mathrm{kG}$, (d) $\mathrm{B}=17.0 \mathrm{kG}$, (e) $\mathrm{B}=28.4 \mathrm{kG}$, (f) $B=56.8 \mathrm{kG} . \quad \mathrm{R}_{\mathrm{c}}=1.85 \mathrm{~cm}$ and $L=5 \mathrm{~cm}$.

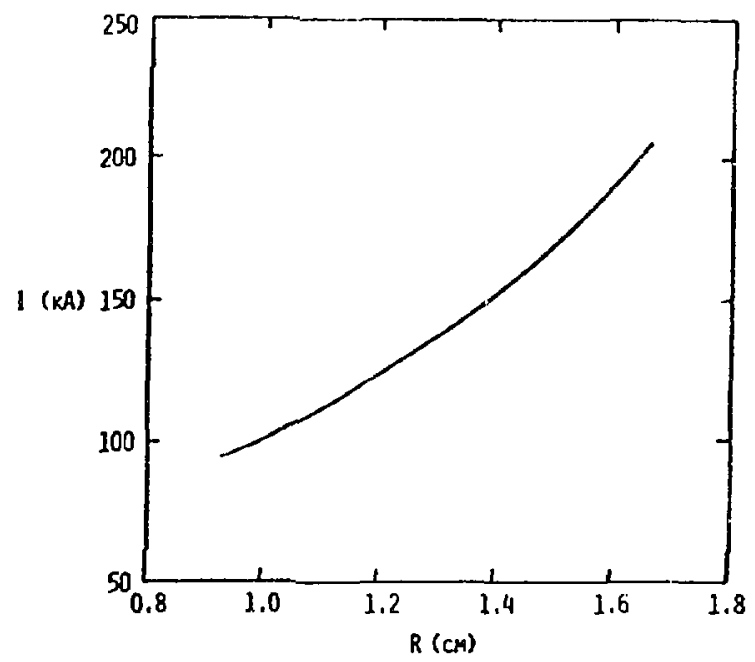

Fig. 4. Tenoni-Proctor theory for space-charge limited current. $R$ is the outer radius of the beam. 


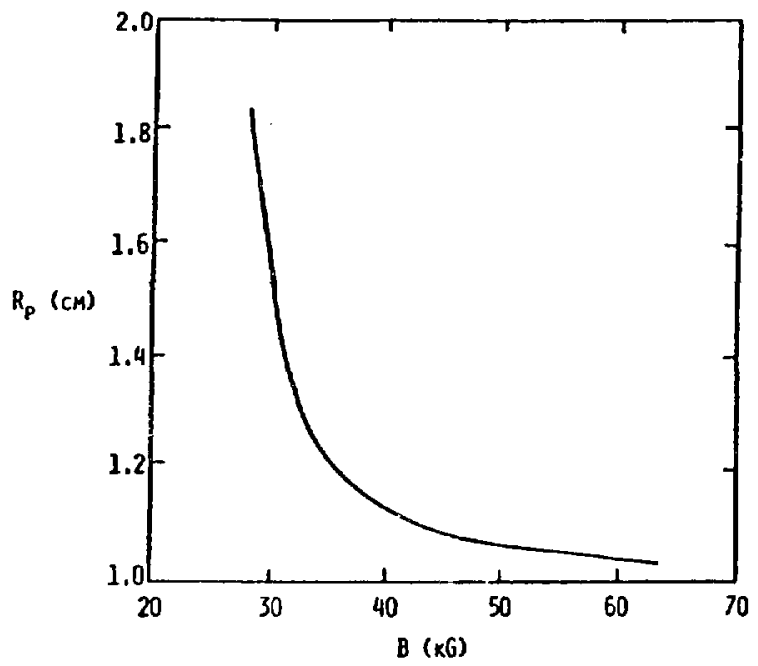

Fig. 5. Outer radius of electron beam as a function of applied magnetic field obtained from

Eq. (7). The parameters

are $I=135 \mathrm{kA}, \gamma=11$, $\mathrm{R}_{\mathrm{o}}=1 \mathrm{~cm}, \mathrm{R}_{\mathrm{C}}=1.85 \mathrm{~cm}$.

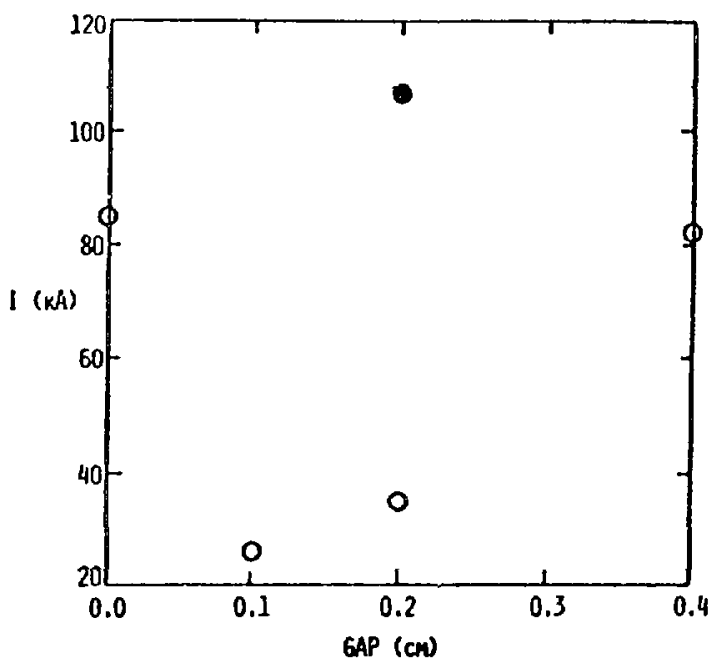

Fig. 7. Diode current versus $A-K$ gap spacing obtained from simulations.

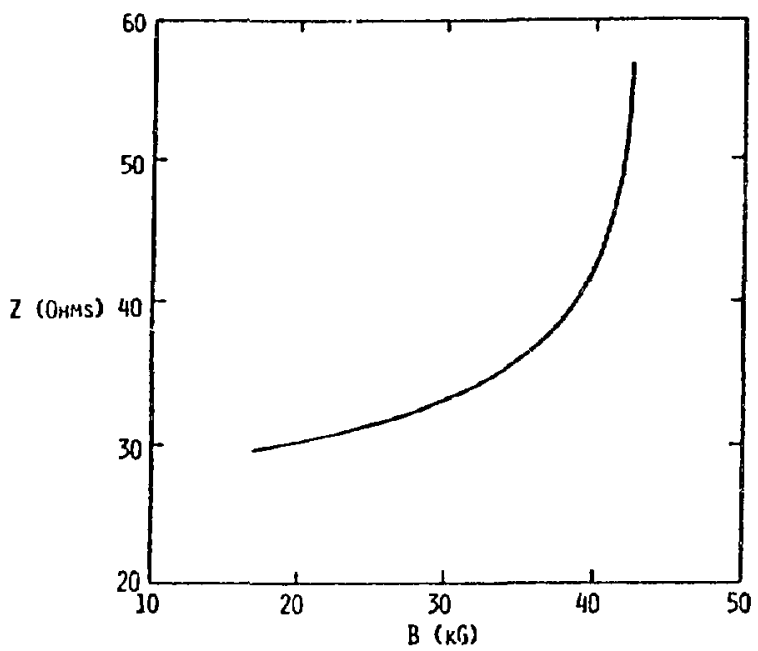

Fig. 6. Impedance of the beam versus magnetic field, for $R=1.2 \mathrm{~cm}$, $R_{0}=1 \mathrm{~cm}, R_{C}=1.85 \mathrm{~cm}$ and $\gamma_{0}^{0}=11$

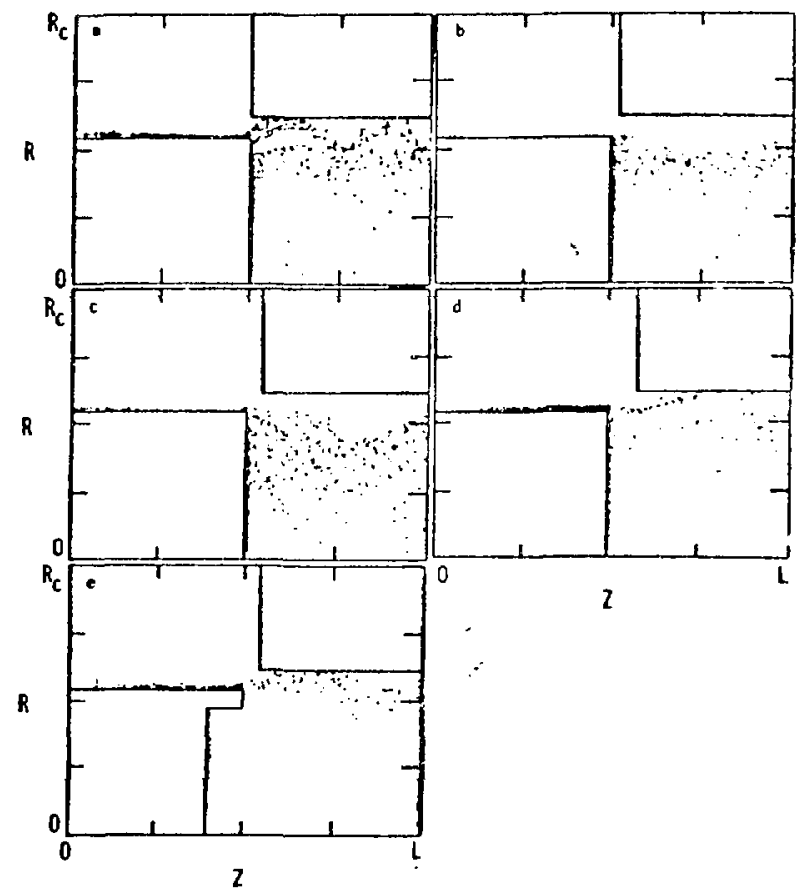

Fig. 8. Equilibrium particle maps of various geometries.

(a) gap $=0$, (b) gap $=0.1 \mathrm{~cm}$,

(c) gap $=0.2 \mathrm{~cm}$, (d) $\mathrm{gap}=0.4 \mathrm{~cm}$,

(e) gap=0.2 cm, shaped cathode. 